forms of torpor; the neuronal and hormonal control of shivering thermogenesis; and the biochemistry of nonshivering thermogenesis.

The assembly of this small group of experts in Prague last year has resulted in the publication of a comprehensive, authoritative and eminently readable series of essays that can be confidently recommended to anyone who needs a relatively painless introduction, or reintroduction, to this exciting field of study.

Although, generally speaking, verbatim reports of the discussions which follow contributions to colloquia are of little value, in this instance I have found the discussions well worth attention. Subsequent reference to this volume will be hampered by the absence of an index. The English is not quite impeccable, but as one who cannot read with confidence in other languages I have nothing but praise for the high standard of Dr Jansky's editorial efforts as well as for his own important contributions to the study of nonshivering thermogenesis.

JOHN BLIGH

\section{Mature Fruit}

The Biochemistry of Fruits and their Products. Vol. 1. Edited by A. C. Hulme. (Food Science and Technology: a Series of Monographs.) Pp. xviii +620 . (Academic: London and New York, November 1970.) £10; $\$ 30$.

As part of a series on food science and technology, the editor quite properly warns that in this book the term "fruit" is to be used in the strictly commercial sense and that, even so, only those fruits most important from an economic stand. point have been chosen. The lessspecialized reader will be encouraged to know that in this first, general volume, the contributors have been allowed to step outside this limit if required by their theme; even Rosa appears here and there.

Again, although the primary study is to be of the maturation, post-harvest physiology, senescence and storage of fruits rather than of their growth processes, a short section on growth and pre-harvest factors is provided. It was indeed here that I found a chapter which seemed to me to be of outstanding merit - "The Physiology and Nutrition of Developing Fruits" by E. G. Bollard. This writer describes, in terms ranging from molecular to morphological, how mature fruits achieve this status, and also their relationship with the parent plant. The reader may profitably begin with this section.

It will already have become obvious that, fortunately, Dr Hulme believes that fruit biochemistry and physiology "cannot usefully be separated". Nor should they be bereft of a background in intracellular and higher levels of structure. One hopes that these latter will find a place in the second volume, which is to present the biochemistry and physiology of individual fruits or types of fruit, and also the biochemistry of fruit processing.

This volume makes a classical approach though the constituents of fruits, with chapters on sugars; hexosans, pentosans and gums; pectic substances; organic acids; amino-acids; proteins ; enzymes; lipids ; volatile compounds; fruit phenolics; carotenoids and triterpenoids, and vitamins. Then comes the section on growth and pre-harvest factors, including hormonal factors, followed by the ethylene factor, the climacteric and ripening. The book ends with a brief consideration of the physiological disorders of fruits.

There are some shortcomings; for example, although chemical constituents are well covered in the subject index, it is less complete in other respects, and when the number of structural formulae in a chapter exceeds seventy the use of Roman numerals is cumbersome. But in general the editor and contributors are to be congratulated. Rarely may the old phrase of "filling a long felt need" be aptly applied: it is well and efficiently earned in this case.

\section{G. A. D. JACKSON}

\section{A Big-bang Universe?}

Modern Cosmology. By D. W. Sciama. Pp. viii $+212+18$ plates. (Cambridge University: London, September 1971.) $£ 3.60 ; \$ 8.95$.

THIS is a survey of the modern developments in cosmology, intended, according to the author, for "readers with only a modest knowledge of mathematics and physics". It is well illustrated with diagrams and photographs and makes interesting reading even for an expert in the field.

The book is divided into sixteen chapters with a theoretical chapter at about the half-way stage on the models of the universe. For the benefit of the non-specialist the first five chapters provide a general description of stars, the Milky-Way system, the external galaxies, radio sources and the quasistellar objects. The associated astrophysical processes such as thermonuclear reactions, cosmic rays, $\mathrm{X}$-rays and $\gamma$-rays, neutrinos, gravitational waves, magnetic fields are also briefly described. The next two chapters discuss the observational data on the distribution of radio galaxies and the quasistellar objects. Then comes the theoretical discussion of the simplest cosmological models. For simplicity there is a brief mathematical discussion which is based on the Newtonian laws of gravitation and motion, in spite of their limited validity. The results from the less ambiguous but more complex theory of relativity are then quoted-and in many ways they are similar to the Newtonian results. With these models in mind the author looks at further astronomical data in the following eight chapters. This largely includes evidence (or lack of it) for the intergalactic matter, the abundance of elements (in particular the helium-hydrogen ratio) and the observed microwave background. The discussion throughout the book is fairly wide but is not deep enough to satisfy the more curious reader. If he wants further information he must look to the more technical reviews available or to the original articles. But, alas, this book does not give references to guide such a reader!

A typical reader is most likely to come to the following conclusions: (1), the redshifts of the quasistellar objects are of cosmological origin; (2), the microwave background is of primaeval (big-bang) origin; and (3), the steady state universe is ruled out by the observations. To what extent are these conclusions justified? One may recall Burbidge's recent remark "that views in cosmology at any epoch are largely determined by the ideas of a few strong individuals, rather than by an objective appraisal of the information available" (Nature, 233, 36 ; 1971). Cosmology has come a long way since Hubble observed the redshift from distant galaxies. As this book shows, the observational astronomer has invented numerous ingenious ways of probing the universe. But the environment has limited the precision of his observations when compared with the precision obtained by a laboratory scientist. This in turn has led to ambiguity of interpretation and to numerous controversies. Cosmology, however, thrives on such controversies; for instance, the three statements given earlier are still subjects of controversy, and should not be taken as facts. The criticism that can be levelled against this book is that it does not make the reader fully aware of the controversial nature of the present data. In an earlier book (The Unity of the Universe, Faber, 1959), Dr Sciama advanced several attractive arguments in support of the steady state theory. Are the present observations really so unequivocal as to warrant a change of view?

Cosmology was (and even now sometimes is) regarded with suspicion by workers in other branches of science for its lack of actual data and its wealth of ingenious theories. It is hoped that by acquainting the sceptic with the very wide scope of observational cosmology today, this book will correct that impression and will thereby serve a useful purpose.

J. V. NARLIKAR 